\title{
Analytic Treatment of Mott-Hubbard Transition in the Half-Filled Hubbard Model
}

\author{
Jongbae Hong and Hae-Young Kee \\ Department of Physics Education, Seoul National University, Seoul 151-742, Korea
}

\begin{abstract}
The Mott-Hubbard transition in the half-filled Hubbard model is studied analytically for the paramagnetic ground state and the classical Néel state. The single-particle density of states is obtained by calculating the Green's function represented by the infinite continued fraction. The paramagnetic solution shows that the Mott-Hubbard transition is signaled by both collapsing Hubbard bands and appearing $\delta$-function peak at midgap, and the transition is second order at the ground state. We also provide specific heats in metallic regime to demonstrate that our results recover those of infinite dimension.
\end{abstract}

PACS numbers: 71.30.+h, 71.10.+x, 75.10.Lp 
The Hubbard model has been studied first by Hubbard[1], Gutzwiller[2], and Kanamori[3] as a model for a narrow band system. It has been recently revisited in relation to a possible model for high-Tc superconductors[4,5]. Besides cuprate superconductors, the Hubbard model may also be applicable to describing the metal-insulator transition(MIT) in the materials like $V_{2-y} O_{3}[6]$ in which paramagnetic insulator, paramagnetic metal, antiferromagnetic insulator, and a new phase antiferromagnetic metal[7] are clearly separated in the temperature-pressure and temperature-filling factor $(y)$ phase diagram. Transitions between these phases[8], mass enhancement[9] due to strong correlation and magnetic ordering $[9,10]$ are common phenomena of the MottHubbard systems such as $\mathrm{Sr}_{1-x} \mathrm{La}_{x} \mathrm{TiO}_{3}[11], \mathrm{Ni}\left(\mathrm{Se}_{1-x} \mathrm{~S}_{x}\right)_{2}[12]$ as well as $\mathrm{V}_{2} \mathrm{O}_{3}$. In addition, an interesting search for a superconducting transition in the metallic $\mathrm{V}_{2} \mathrm{O}_{3}$ is suggested in close relation to a Mott-Hubbard insulator shown in the rare-earth oxide superconductors and the strong electron correlation shown in the heavy-fermion superconductors.[7] These bring renewed interest in the Mott-Hubbard transition(MHT), recently.

The MHT accompanies both electronic and magnetic transitions. Pure $\mathrm{V}_{2} \mathrm{O}_{3}$ in a paramagnetic metal phase becomes antiferromagnetic insulator through the antiferromagnetic ordering of the vanadium spins at a temperature around 150K.[6] Above this temperature, paramagnetic metal-insulator transition occurs. This transition temperature, however, can be suppressed until very low temperature by applying hydrostatic pressure, adjusting the 
oxygen stoichiometry, and alloying with titanium sesquioxide.[7-9] Therefore, both paramagnetic metal-insulator transition due to strong correlation and paramagnetic metal-antiferromagnetic insulator transition due to antiferromagnetic ordering are interesting. These transitions accompany Fermi liquid quasiparticle mass enhancement through the strong electron correlations, which has been studied by many experiments[8,9,11]. On the other hand, no successful theories exist to explain the experimental results except the large-d approach[12-15] as far as we know.

The beauty of large-d approach lies in the ability to solve the Hubbard model, in principle, exactly in a well-defined limit in which spatial correlations do not play an important role. Unfortunately, some degree of insight into the local dynamics contributing to the end-results is concealed as a component of the numerical self-consistency that is an integral part of this approach. In an effort to compliment this procedure, in this Letter we introduce an extremely simple approach to the dynamics of the MHT by calculating the single-particle density of states(DOS) through a continued fraction formalism for the Green's function. The formalism is known as projection operator technique[16], recurrence relation method[17], and dynamical Lanzcos method[5]. The method that we use has been previously applied with great success to the response function of the electron gas at a metallic density at which usual perturbation technique may not be that successful.[18] From the study on the electron gas, we found that infinite continued fraction provides 
analytic expression for the dynamics of the electron system by using minimal amount of information about the ground-state correlations.

Here we study the MHT on a bipartite lattice for the paramagnetic ground state and the classical Néel state. The paramagnetic metal-insulator transition is realized when temperature is above the Néel temperature. Below the Néel temperature, however, the MHT does not occur at a finite onsite Coulomb interaction $U$ on a bipartite lattice because of appearing antiferromagnetic long-range order due to perfect nesting.[19] Even though the paramagnetic metal-insulator transition is realized only above the Néel temperature, the physics of the transition in the artificial paramagnetic ground state, for instance, the Brinkman-Rice scenario[20], is also interesting to understand the strong correlation effect in the MHT in the Hubbard model. This problem, especially in a finite dimension, has been considered as one of quite difficult tasks in condensed matter physics, because one must treat both spatial correlations and local fluctuations equally rigorously.

As a lot of insight is gained by reproducing complicated results within a very simple theory, in this work we show that a very large fraction of the essential physics described in the large-d approach such as the Hubbard bands, the quasi-particle peak, and the second order feature of the MHT at ground state can be reproduced by a simple continued fraction expansion about atomic state. This reproduction may be understood by the arguments advanced as part of the large-d approach that a great deal of physics in the 
MHT is dominated by local dynamics.[12-14]

As for a finite dimension, however, no first principles calculation has been done to obtain the single-particle DOS even for the atomic state as far as we know. But our analytic solution keeps the dimensional dependence even though approximation becomes cruder as one goes to a lower dimension. The effect of spatial correlation which brings more prominent dimensional dependence to the DOS will be studied in the future.

In this Letter, we first show that the classical Néel state which is close to the real ground state of the half-filled Hubbard model yields Hubbard gap for any $U>0$ in the single-particle DOS. Thus we recover a well-known fact that the MHT occurs at $U=0$ for the Hubbard model on a bipartite lattice. The paramagnetic solution for the DOS shows the Hubbard feature[1] and the Brinkman-Rice feature[20] for the MHT simultaneously in a very simple analytic form. We also show that the thermodynamics obtained by this DOS reproduces that of large-d, which confirms the similarity of the approximations used in the large-d approach and our simple method.

The single-particle DOS is obtained by calculating the one-particle Green's function of the fermion operator at the same site, i.e., $\left\langle\Psi_{0}\left|\left\{c_{j \sigma}^{\dagger}(t), c_{j \sigma}\right\}\right| \Psi_{0}\right\rangle$ where $c_{j \sigma}^{\dagger}$ and $c_{j \sigma}$ are fermion creation and annihilation operators with spin $\sigma$ at site $j$, curly brackets means anticommutator, and $\left|\Psi_{0}\right\rangle$ denotes ground 
state. The $\operatorname{DOS} \rho_{\sigma}(\omega)$ is given by $[1]$

$$
\rho_{\sigma}(\omega)=\frac{2}{N} \lim _{\epsilon \rightarrow 0^{+}} \sum_{j} \operatorname{Im} G_{j j}^{(-)}(\omega-i \epsilon),
$$

where

$$
\begin{aligned}
G_{j j}^{(-)}(\omega-i \epsilon) & =\frac{i}{2 \pi} \int_{0}^{\infty}\left\langle\Psi_{0}\left|\left\{c_{j \sigma}^{\dagger}(t), c_{j \sigma}\right\}\right| \Psi_{0}\right\rangle e^{-i \omega t-\epsilon t} d t \\
& =\frac{1}{2 \pi}\left\langle\Psi_{0}\left|\left\{c_{j \sigma},(\omega-L-i \epsilon)^{-1} c_{j \sigma}^{\dagger}\right\}\right| \Psi_{0}\right\rangle \\
& \left.\equiv \frac{i}{2 \pi} a_{0}(z)\right|_{z=i \omega+\epsilon},
\end{aligned}
$$

where $L$ is the Liouville operator. The superscript (-) denotes usual notation of the advanced Green's function. The Green's function (2) may be represented by an infinite continued fraction $[5,16,17]$. Thus the single-particle DOS is written as

$$
\rho_{\sigma}(\omega)=\left.\frac{1}{\pi N} \lim _{\epsilon \rightarrow 0^{+}} \sum_{j} \operatorname{Re} a_{0}(z)\right|_{z=i \omega+\epsilon},
$$

where

$$
a_{0}(z)=\frac{1}{z-\alpha_{0}+\frac{\Delta_{1}}{z-\alpha_{1}+\frac{\Delta_{2}}{z-\alpha_{2}+} \cdot}},
$$

where $\alpha_{\nu}=\left(i L f_{\nu}, f_{\nu}\right) /\left(f_{\nu}, f_{\nu}\right), \Delta_{\nu}=\left(f_{\nu}, f_{\nu}\right) /\left(f_{\nu-1}, f_{\nu-1}\right)$. The inner product is defined by $(A, B)=\left\langle\Psi_{0}\left|\left\{A, B^{\dagger}\right\}\right| \Psi_{0}\right\rangle$ where $A$ and $B$ are operators of the Liouville space, $B^{\dagger}$ is the adjoint of $B$.

The Hubbard model which we study here is written as

$$
H=-\sum_{<j l>\sigma} t_{j l} c_{j \sigma}^{\dagger} c_{l \sigma}+\frac{U}{2} \sum_{j \sigma} n_{j \sigma} n_{j,-\sigma}
$$


where $\left\langle j l>\right.$ means nearest neighbor sites. To obtain the DOS $\rho_{\sigma}(\omega)$ where $\sigma$ denotes spin-up state, we choose $f_{0}=c_{j \sigma}^{\dagger}$ and calculate $\alpha_{\nu}$ and $\Delta_{\nu}$ by using the recurrence relation[17]

$$
f_{\nu+1}=i L f_{\nu}-\alpha_{\nu} f_{\nu}+\Delta_{\nu} f_{\nu-1}
$$

The key approximation in this process is the Hartree-Fock approximation in calculating the ground state correlation function such as $\left\langle n_{j,-\sigma} n_{l,-\sigma}\right\rangle=$ $\left\langle n_{j,-\sigma}\right\rangle\left\langle n_{l,-\sigma}\right\rangle$. This corresponds to setting the ground state $\left|\Psi_{0}\right\rangle$ as a product of atomic states and to neglecting spatial correlations which is valid at large-d limit. A similar decoupling scheme has been done on the average containing the operator $\left[H, n_{j \sigma}\right]$ whose average vanishes, since no steady current exists in the ground state.

First we obtain $\rho_{\sigma}(\omega)$ for the classical Néel state to study the MHT for a state with antiferromagnetic long-range order. We take antiferromagnetic spin configuration such that the spin at site $j$ is $\downarrow$. Then we obtain

$$
\begin{aligned}
\alpha_{\nu} & =i U \text { when } \nu \text { is odd, } \\
& =0 \text { when } \nu \text { is even, }
\end{aligned}
$$

and

$$
\Delta_{1}=q t^{2}, \quad \Delta_{\nu}=(q-1) t^{2} \text { for } \nu \geq 2
$$

To obtain $\rho_{-\sigma}(\omega)$, on the other hand, we choose $f_{0}=c_{j,-\sigma}^{\dagger}$. Then we obtain $\alpha_{\nu}=0$ for odd $\nu$ and $i U$ for even $\nu$ and have the same $\Delta_{\nu}$ as above. Using 
these, $\rho(\omega)=\rho_{\sigma}(\omega)+\rho_{-\sigma}(\omega)$ is given by

$$
\rho(\omega)=\frac{\frac{4}{\pi} q(q-1) t^{4}|\omega| \sqrt{\left(\omega^{2}-\frac{U^{2}}{4}\right)\left(\frac{U^{2}+16(q-1) t^{2}}{4}-\omega^{2}\right)}}{(q-2)^{2}\left(\omega^{2}-\frac{U^{2}}{4}\right)^{2}+q^{2} t^{4}\left(\omega^{2}-\frac{U^{2}}{4}\right)\left(\frac{U^{2}+16(q-1) t^{2}}{4}-\omega^{2}\right)}
$$

Eq. (9) satisfies sum rule exactly and yields Hubbard gap which makes the system insulator for any $U>0$. We plot Eq. (9) for $U=0$ and $U=1 t$ for $q=4$ in Fig. 1, for instance.

For the paramagnetic ground state in which one can see the MHT at finite $U$, we obtain the following regular patterns for $\Delta_{\nu}$ and $\alpha_{\nu}$ for large- $U$ :

$$
\begin{aligned}
\Delta_{1} & =\frac{U^{2}}{4}+q t^{2}, \\
\Delta_{2} & =\frac{\frac{1}{2} U^{2} q t^{2}+q(q-1) t^{4}}{\frac{1}{4} U^{2}+q t^{2}}, \\
\Delta_{2 \nu+1} & =\frac{U^{2}}{4}\left\{1+O\left(\frac{t^{2}}{U^{2}}\right)\right\} \equiv a, \\
\Delta_{2 \nu+2} & =4 q t^{2}\left\{1+O\left(\frac{t^{2}}{U^{2}}\right)\right\} \equiv b,
\end{aligned}
$$

where $\nu \geq 1$ and $\alpha_{0}=\alpha_{1}=\alpha_{2}=i U / 2$ and $\alpha_{\nu}=i \frac{U}{2}\left\{1+O\left(t^{2} / U^{2}\right)\right\}$ for $\nu \geq 3$. Only even powers of $t / U$ appear in the expansion for $\Delta_{\nu}$ and $\alpha_{\nu}$ for $\nu \geq 3$. A further approximation $(q-1) \approx q$ which is valid at higher dimension has also been made.

By neglecting terms of $O\left(t^{2} / U^{2}\right)$, the infinite continued fraction (4) can be easily calculated, and the result is

$$
a_{0}(\tilde{z})=\frac{\tilde{z}+\frac{\Delta_{2}}{2 b}\left[\frac{(b-a)}{\tilde{z}}-\tilde{z}+\frac{1}{\tilde{z}} \sqrt{\left(\tilde{z}^{2}+a-b\right)^{2}+4 b \tilde{z}^{2}}\right]}{\tilde{z}^{2}+\frac{\Delta_{2}}{2 b}\left[(b-a)-\tilde{z}^{2}+\sqrt{\left(\tilde{z}^{2}+a-b\right)^{2}+4 b \tilde{z}^{2}}\right]+\Delta_{1}},
$$


where $\tilde{z}=z-\alpha_{0}$. We choose positive sign in front of the square root instead of negative sign to satisfy the boundary condition $a_{0}(t=0)=1$, where $a_{0}(t)$ is the inverse Laplace transform of $a_{0}(\tilde{z})$.

If one sets the chemical potential at $\mu=\frac{U}{2}$, Eq. (3) gives the DOS as follows:

$$
\begin{aligned}
\rho_{\sigma}(\omega)= & \frac{\frac{\Delta_{1} \Delta_{2}}{2 b \pi|\omega|} \sqrt{W}}{\left[\frac{\Delta_{2}}{2 b}(b-a)+\Delta_{1}+\left(\frac{\Delta_{2}}{2 b}-1\right) \omega^{2}\right]^{2}+\left[\frac{\Delta_{2}^{2}}{4 b^{2}} W\right]} \text { for } \mathrm{a}>\mathrm{b} \\
= & \frac{\left(1-\frac{a}{b}\right) \delta(\omega)}{\frac{\Delta_{1}}{\Delta_{2}}+\left(1-\frac{a}{b}\right)}+ \\
& \frac{\frac{\Delta_{1} \Delta_{2}}{2 b \pi|\omega|} \sqrt{W}}{\left[\frac{\Delta_{2}}{2 b}(b-a)+\Delta_{1}+\left(\frac{\Delta_{2}}{2 b}-1\right) \omega^{2}\right]^{2}+\left[\frac{\Delta_{2}^{2}}{4 b^{2}} W\right]} \text { for } \mathrm{a}<\mathrm{b},
\end{aligned}
$$

where $W=\left\{\omega^{2}-(\sqrt{a}-\sqrt{b})^{2}\right\}\left\{(\sqrt{a}+\sqrt{b})^{2}-\omega^{2}\right\}$. From the single-particle DOS, one can see that MIT occurs at $a=b$, i.e., $U_{c}=4 \sqrt{q} t$ and the Fermi liquid quasi-particle mode appears at midgap in metallic phase, i.e., $U<U_{c}$ and the Hubbard bands have band width $4 \sqrt{q} t$ in insulator $(a>b)$ and $U$ in metallic phase $(a<b)$. The band gap is $|U-4 \sqrt{q} t|$. To draw $\rho_{\sigma}(\omega)$ in the large-d limit, we scale $t$ as $t=t_{*} / \sqrt{2 q}$ and set $t_{*}=1$. We plot three typical cases, i.e., (a) $U=4$ (two bands), (b) $U=U_{c}=2 \sqrt{2}$ (single band), (c) $U=2.4$ (two bands with $\delta$-function peak at $\omega=0)$ in Fig. 2.

The $\delta$-function quasi-particle peak at zero frequency is of course an artifact, and a more realistic calculation would lead to a dispersive band. In fact, a similar calculation to present one but expanded for small- $U$ shows the dispersive band, which will be submitted in a separated paper. Nevertheless, 
the weight of the $\delta$-function peak gives us a measure of the quasi-particle mass renormalization $Z_{F}=m / m^{*}$. The quasi-particle mass diverges as $U$ approaches $U_{c}$ from metallic side. The Brinkman-Rice scenario for MIT[20] can be seen here.

It is interesting to compare our results to those of large-d. Our DOS shows that the MHT in paramagnetic ground state is second order, which coincides with the recent results[15] of infinite dimension. The dispersive band shown in the large-d results may be reproduced by applying the Luttinger theorem[21] which fixes the height of the quasi-particle peak to the value of the DOS at Fermi energy of the noninteracting system. For further comparison with the large-d approach, we extract a part of thermodynamics from the DOS (12).

The extension of our formalism to the finite temperature is rather simple, since the temperature-dependence only enters in the correlation function such as $\left\langle n_{j-\sigma} n_{l-\sigma}\right\rangle$ which vanishes within Hartree-Fock approximation. Therefore, the temperature-dependence does not appear in the DOS in atomic limit. Thus the internal energy has temperature- dependence only in the Fermi distribution function $f(\omega)$. In the metallic regime, the internal energy is given by

$$
E(T)=\int_{-\infty}^{\infty} \omega f(\omega)\left\{\rho_{i n c}(\omega)+\rho_{q p}(\omega)\right\} d \omega
$$

where $\rho_{\text {inc }}(\omega)$ is the incoherent part of Eq. (12) and $\rho_{q p}(\omega)$ is the quasiparticle DOS. The latter is the coherent part of Eq. (12) divided by the 
quasi-particle mass renormalization $Z_{F}$, which can be written as

$$
\rho_{q p}(\omega)=\frac{1}{\pi Z_{F}} \frac{\eta}{\omega^{2}+\eta^{2}}
$$

The parameter $\eta$ is determined by the condition $\rho_{q p}(0)=N(0) / Z_{F}$, where $N(0)=\sqrt{2} / \pi[22]$. The specific heats calculated from Eq. (13) for $U$ 's which are a little bit less than $U_{c}$ are shown in Fig. 3. One can see a peak at low-temperature regime which is given by the Fermi liquid quasi-particle.

One of interesting features which looks peculiar among the results of large-d approach is shown in the specific heat at metallic regime $\left(U<U_{c}\right)$. The specific heats for different U's show a crossing at a certain temperature, $T \approx 6.5$. This phenomenon has also been shown in the experiments for ${ }^{3} \mathrm{He}[23]$. But the physical origin of it is not clear yet. These phenomena are the characteristics of large-d result[8]. Other thermodynamic quantities obtained from Eqs. (11) and (12) will be appeared in the forthcoming paper.

As final remarks, we mention about our approximation. The HartreeFock approximation in calculating the static correlation functions is the only approximation when we obtain the DOS for the classical Néel state. For the paramagnetic solution, however, we neglect $O\left(t^{2} / U^{2}\right)$ terms compared with unity in obtaining $\Delta_{\nu}$ and $\alpha_{\nu}$ for $\nu \geq 3$ in addition to the Hartree-Fock approximation. The neglected terms may shifts the positions of poles a little bit when they are included. We imagine that this effect may not change the over-all physics contained in the DOS. Even under these approximation, our 
DOS satisfies sum rule exactly, i.e. $\int_{-\infty}^{\infty} \rho_{\sigma}(\omega) d \omega=1$, for $U$ 's given in Fig. 2 .

The underlying reason for the remarkable agreement between the large$\mathrm{d}$ and the simple continued fraction method must lie in the fact that the physics of the large-d limit is dominated by the atomic limit. The continued fraction expansion used here may provide the possibility to treat the effects of quantum fluctuation in the classical Néel state and spatial correlation as well as doping. These will be the subjects of forthcoming paper.

The authors wish to express their gratitude to the International Center for Theoretical Physics for financial support and hospitality. They also thank Prof. P. Coleman, Prof. P. Fazekas for useful discussions and Prof. Yu Lu for encouragement. One of authors(J.H.) also thanks the Government of Japan for support. This work has been supported by SNU-CTP and Basic Science Research Institute Program, Ministry of Education. 
[1] J. Hubbard, Proc. Roy. Soc. A276, 238 (1963) and A281, 401 (1964).

[2] M. Gutzwiller, Phys. Rev. Lett. 10, 159 (1963).

[3] J. Kanamori, Prog. Theor. Phys. 30, 275 (1963).

[4] P. W. Anderson, Science 235, 1196 (1987).

[5] E. Dagotto, Rev. Mod. Phys. 66, 763 (1994).

[6] D. B. McWhan, A. Menth, J. P. Remeika, W. F. Brinkman, and T. M. Rice, Phys. Rev. B 7, 1920 (1973).

[7] S. A. Carter, T. F. Rosenbaum, J. M. Honig, and J. Spalek, Phys. Rev. Lett. 67, 3440 (1991).

[8] S. A. Carter et al., Phys. Rev. B 43, 607 (1991).

[9] S. A. Carter et al., Phys. Rev. B 48, 16841 (1993).

[10] W. Bao et al., Phys. Rev. Lett. 71, 766 (1993).

[11] Y. Tokura Phys. Rev. Lett. 70, 2126 (1993).

[12] A. Georges and G. Kotliar, Phys. Rev. B 45, 6479 (1992).

[13] A. Georges and W. Krauth, Phys. Rev. B 48, 7167 (1993).

[14] M. J. Rozenberg, G. Kotliar, and X. Y. Zhang, Phys. Rev. B 49, 10181 (1994).

[15] M. Caffarel and W. Krauth, Phys. Rev. Lett. 72, 1545 (1994); M. J. Rozenberg, G. Moeller, and G. Kotliar, SISSA \#cond-mat/9402056

[16] H. Mori, Prog. Theor. Phys. 33, 423 (1965), and 34, 399 (1965); P. Fulde, Electron Correlations in Molecules and Solids Solid-State Sciences Vol. 100 (Springer-Verlag, Berlin, 1993). 
[17] J. Hong, J. Kor. Phys. Soc. 20, 174 (1987); M. H. Lee, J. Math. Phys. 24, 2512 (1983), Phys. Rev. Lett. 49, 1072 (1982), and Phys. Rev. B26 (1982).

[18] J. Hong and M. H. Lee, Phys. Rev. Lett. 70, 1972 (1993); 55, 2375 (1985).

[19] J. C. Slater, Phys. Rev. 82, 538 (1951); J. E. Hirsch and S. Tang, Phys. Rev. Lett. 62, 591 (1989).

[20] W. F. Brinkman and T. M. Rice, Phys. Rev. B 2, 4302 (1970).

[21] E. Muller-Hartmann, Z. Phys. B 74, 507 (1989).

[22] The continued fraction (4) for small- $U$ limit gives $\rho_{\sigma}(0)=\sqrt{q-1} / \pi q t$ which corresponds to $\sqrt{2} / \pi t_{*}$.

[23] D. S. Greywall, Phys. Rev. B 27, 2747 (1983). 


\section{Figure Captions}

Fig. 1 : The single-particle density of states $\rho(\omega)$ for the classical Néel state. The dotted line is for $U=0$ and the solid line for $U=1 t$.

Fig. 2 : The single-particle density of states $\rho_{\sigma}(\omega)$ of the paramagnetic phase for large-d limit. (a) $U=4 t_{*}$, (b) $U=U_{c}=2 \sqrt{2} t_{*}$, (c) $U=2.4 t_{*}$. The number $(0.11)$ in (c) represents the weight of $\delta$-function.

Fig. 3 : The specific heats for $U<U_{c}=2 \sqrt{2}$. The solid, dashed, and dotted lines are for $U=2.6,2.4$, and 2.2 , respectively. We set $t_{*}=1$. 